\title{
La idea de heurística en estudiantes para profesor de matemáticas
}

\author{
Recibido: 13-05-2010| Aceptado: 30-06-2011
}

The heuristic idea of mathematics pre service teachers

Alvaro Arturo Sanjuan*

Edna Paola Fresneda**

Aseneth Gutiérrez ${ }^{* *}$

Oscar Leonardo Pantano**
Resumen: Este artículo es el resultado de una coinvestigación enmarcada en la Investigación «El proceso de demostración como instrumento de aprendizaje en la formación de profesores», desarrollada por el Grupo MESCUD de la Universidad Distrital Francisco José de Caldas. La investigación gira en torno al aprendizaje de la demostración en los estudiantes para profesor de matemáticas en un contexto matemático de continuidad de reales. Se tiene en cuenta la perspectiva de la demostración que explica en dónde los estudiantes emplean representaciones, ejemplos y argumentaciones propias.

La metodología empleada consistió en un experimento de enseñanza como una forma de la investigación formativa. Se enfoca en el perfeccionamiento progresivo de un diseño basado en la puesta en práctica del mismo. Este diseño es constantemente revisado y modificado, por el equipo de investigadores, con el fin de mejorar el aprendizaje. El análisis de los datos se sustenta en la técnica de las viñetas; es decir, informes que integran información de diferentes fuentes de la práctica del profesor, interpretaciones e inferencias del investigador.

Así, se vincula, desde el quehacer matemático de los estudiantes, la evidencia de cómo el uso de diversas estrategias heurísticas en el proceso de resolución permite comprender la demostración. A su vez, estas estrategias y procedimientos posibilitan la concepción de la demostración como el objetivo principal de su proceso de resolución, dándole sentido a la actividad del estudiante.

Palabras clave: Toma de conciencia, heurísticas, resolución de problemas, comunidad de aprendizaje, metacognición, objetivación.

\begin{abstract}
This article is a result of a research framed in the Research study "The demonstration process as a learning tool in teacher Education" developed by the Research Group MESCUD at Universidad Distrital Francisco José de Caldas. The research is focused on demonstration learning with pre service mathematics students in a course of real continuity. It takes into account the perspective of the experimentation where students use representations, examples and arguments from their own.

The methodology consisted of a teaching experiment as a form of formative research. They focus on the progressive refinement of a design based on its implementation. This design is constantly reviewed and modified by the research team, in order to enhance learning. The data analysis technique is based on the bullets, that is, reports that integrate information from different sources of the practice teacher's interpretations and researcher's inferences.

Thus the evidence of how the use of various heuristic strategies in the resolution process led to the understanding of experimentation is linked to the students' mathematical task. In turn, these strategies and procedures enable the conception of experimentation as the main objective of the process of resolution, giving meaning to student activity.
\end{abstract}

Keywords: Awareness, heuristics, problem solving, community learning, metacognition, objectification.

* Profesor de la Universidad Distrital Francisco José de Caldas

* Estudiantes Licenciatura en Educación Básica con Énfasis en Matemáticas Universidad Distrital. aasanjuanc@udistrital.edu.co, epfresnedap@estudiante.udistrital.edu.co, fagutierrezr@estudiante.udistrital.edu.co, olpantanom@estudiante.udistrital.edu.co 


\section{Introducción}

El aprendizaje de la demostración ha sido ampliamente discutido en la comunidad de investigadores en educación matemática. Existen, al menos, tres enfoques claramente diferenciados. El enfoque constructivista que considera el aprendizaje de la demostración como un proceso, más o menos continuo, mediado por la interacción y las normas sociomatemáticas instaladas en la clase (Simon, 1996). En este enfoque se privilegia una forma de argumentación en matemáticas basado en la demostración que explica Hanna (1990). La demostración que explica son todos aquellos argumentos matemáticos del estudiante soportados en su actividad de resolución, es decir, las representaciones y los ejemplos propios.

Por su parte, el enfoque cognitivo tiene en cuenta los procesos de representación involucrados en el proceso de aprendizaje (Duval, 1991). Aquí, el aprendizaje de la demostración se soporta en un análisis funcional de la lengua y los sistemas de representación semiótica. Por último, el enfoque sociocultural, más reciente, que involucra el aprendizaje en la práctica; donde se considera deseable que la tematización de la demostración se haga de manera explícita y de manera implícita (Hemmi, 2008).

El aprendizaje de la demostración en esta investigación implicó el uso de heurísticas. Desde una perspectiva sociocultural, el aprendizaje de la demostración debe llevar una práctica implícita y una práctica explicita (Hemmi, 2008). Partimos de dos hipótesis; por un lado, que es deseable que el conocimiento profesional de los profesores de matemáticas involucre las heurísticas (Puig, 1996) y por otra parte, que el aprendizaje de las heurísticas también debe tener una práctica implícita.
Así, en este trabajo pretendemos discutir, desde la perspectiva sociocultural del aprendizaje fundamentada en la teoría cultural la objetivación, cómo es el proceso de toma de conciencia de los estudiantes para profesor de matemáticas de la idea de heurística; anclada a procesos de demostraciones que explican.

\section{Elementos Teóricos}

\section{Teoría cultural de la objetivación}

Una manera de entender la resolución de problemas en el aula de matemáticas, es cuando el objetivo del aprendizaje busca que el estudiante imite una práctica establecida. Cuando esto ocurre Radford (2006, p. 114) lo denomina "aprender a hacer en matemáticas". Ahora bien, cuando la resolución de problemas no es el fin, sino el medio para alcanzar la reflexión cultural en la que se forman las capacidades humanas de los individuos, Radford (p. 114) lo denota: "aprender a ser en matemáticas". De este modo "El aprendizaje no consiste en construir o reconstruir un conocimiento sino en dotar de sentido a los objetos conceptuales que encuentra el alumno en su cultura" (p. 113).

Para desarrollar la idea de aprendizaje, enmarcada en la teoría cultural de la objetivación, el pensamiento es la base del aprendizaje, pues a través de éste se establece la relación entre el ser y el mundo. En este enfoque se discute que "El proceso real del pensamiento queda invisible así como los conceptos que éste usa y el material crudo del cual está compuesto" (p. 105) y en contraste el pensamiento, base del aprendizaje, es completamente dependiente de recursos culturales para su propia operación, es decir, una práctica social (algo que se aprende haciendo). De esta manera, la idea de pensar asume una realidad ontológica (la existencia y la 
La idea de heurística en estudiantes para profesor de matemáticas Alvaro Arturo Sanjuan | Edna Paola Fresneda | Aseneth Gutiérrez | Oscar Leonardo Pantano

naturaleza del objeto). Los objetos matemáticos son construidos a lo largo de la historia producto de la actividad matemática de los individuos. En otras palabras, "Los objetos matemáticos son patrones fijos de actividad reflexiva incrustados en el mundo en cambio constante de la práctica social mediatizada por los artefactos" (p. 111).

De acuerdo con lo anterior, la relación entre el objeto y el sujeto es concebida como la re-flexión de este último sobre el mundo que lo rodea. Sin embargo, cabe aclarar que esta re-flexión no es un acto interno y difiere de la reflexión llamada a menudo metacognición. Pues esta última, "no es otra cosa que la atención a aquello que ya está en nosotros" (p. 123).

A su vez, ser en matemáticas exige un proceso de elaboración de significados, para éste la adquisición del saber se desarrolla por medio del contacto del sujeto con dos fuentes distintas. Los artefactos y la interacción social.

Artefactos. En cuanto al contacto del sujeto con los artefactos, plano sujeto-objeto ( $p$. 124), los artefactos se caracterizan por potenciar la cognición humana. Dado que llevan depositada en sí la sabiduría histórica de la actividad cognitiva de generaciones pasadas. Así, la actividad del sujeto se ve orientada y moldeada por los artefactos.

Interacción social. Allí se resalta la sociabilidad, proceso que permite aprender a estar con otros, a escuchar y entender otras voces y otras conciencias. Así, la interacción social, concebida desde la teoría cultural de la objetivación, es semejante a los planteamientos de la Teoría de las comunidades de práctica expuestos por Wenger (2001) que en los contextos institucionales de aprendizaje reciben el nombre de comunidades de aprendizaje.
Comunidades de aprendizaje. Esta teoría se fundamenta en la interacción constante de un grupo de estudiantes en torno a una empresa. En el contexto escolar, la empresa puede ser la resolución de un problema de matemáticas. Así, para conseguir dicho objetivo se evidencian procesos de diálogo, colaboración, participación y negociación de significados.

Además, el aprendizaje aparece como una estructura social susceptible al cambio pero que debe tener un carácter de estabilidad. De este modo, cuando la comunidad se encuentra en desestabilidad, siente la necesidad de adaptarse a las nuevas condiciones que exige el aprendizaje. Resultado de éste, se produce el significado, fruto de las interacciones con los elementos inmersos en el mundo.

En primer lugar, la participación se refiere a una interacción en comunidades sociales, dada por la experiencia con los objetos que se encuentran en la cultura (artefactos). Ésta se entiende de forma individual y colectiva, dando cuenta de nuestra experiencia social de vivir en el mundo. Por su parte, la cosificación da forma a nuestra experiencia plasmando el objeto de aprendizaje en una cosa. Esto permite comprenderlo mejor y cambia nuestra relación con éste, contribuyendo a dotarlo de significado. Así, la cosificación hace referencia tanto al proceso como al producto de dicho proceso.

El proceso de objetivación y toma de conciencia. El proceso de objetivación hace referencia a la re-flexión sobre aquellos objetos que se encuentran en la cultura. Ésta posibilita el encuentro entre el sujeto y el objeto del saber mediatizados, que se caracteriza por ser un proceso social y no individual. Dicha sociabilidad implica el proceso de formación de la conciencia, es decir, un saber común que se construye a través de la actividad del individuo. 
Esta actividad se convierte en un movimiento progresivo hacía el objeto, es decir, en un proceso en el que se evidencia inicialmente que el objeto está formado por elementos de naturalezas diversas, que nos permiten concebirlo y pensarlo desde diferentes representaciones. Lo anterior, dado que los objetos han sido expresados de diversas formas a lo largo de la historia, cada una de las cuales ofrece un significado distinto.

Aún así, dichos elementos o formas de pensar el objeto apuntan solamente a éste de acuerdo con las características que le han sido otorgadas desde la cultura. En el proceso cognitivo, tales elementos o formas de pensar el objeto son percibidos de manera gradual y progresiva. Cuando este proceso se ha desarrollado en su totalidad podemos decir que el sujeto ha notado el objeto por medio de su acción.

En consecuencia, la toma de conciencia del objeto es un proceso en el que objeto y sujeto, tan distintos en su naturaleza, dialogan mediados por la interacción social y los artefactos. Esto es lo que en últimas, desde esta perspectiva, es aprender. En otras palabras, el aprendizaje es un proceso dialéctico entre sujeto y objeto, donde el primero por medio de su acción abstrae la generalidad y el sentido del segundo, de manera progresiva (p. 115).

\section{Resolución de problemas y uso de heurísticas}

Resolución de problemas. La resolución de problemas es concebida como una forma de pensar que les permite a los estudiantes desarrollar habilidades creando y utilizando diversas estrategias (Santos, 2007). Lo que no sería posible si éstos no generan cuestionamientos sobre la resolución de problemas y los elementos inmersos en dicho proceso.

Partiendo de estas ideas el aprendizaje de las matemáticas se fundamenta en la construcción y el desarrollo de relaciones que enfrentan al estudiante con las actividades realizadas por un matemático (p. 17). Allí aparecen elementos constitutivos de dicha ciencia a los cuales se enfrenta llevando a cabo acciones que le permiten dotarlos de sentido.

A su vez Puig (1996) menciona que el proceso de resolución de problemas inicia en el momento en el que el individuo reconoce que lo que se tiene allí es un problema y que debe resolverse. Así, durante este proceso el resolutor lleva a cabo actividades mentales $y$ acciones que le permiten enfrentarse al problema que se le plantea.

Heurísticas. Según Puig (1996), lo que es propio de la heurística es el estudio de los modos de comportamiento y los medios que se utilizan al resolver problemas, esto no supone garantía de que se obtenga solución. Este autor clasificó las heurísticas en tres grupos: herramientas heurísticas, destrezas heurísticas y sugerencias heurísticas. Aunque son de naturaleza distinta en el proceso de resolución de problemas estas heurísticas pueden combinarse. El uso de una de éstas puede conllevar a la utilización de otra.

Las herramientas heurísticas son aquellas que transforman el problema original en otro diferente. Básicamente, son aquellos elementos de transformación, los cuales son independientes del contenido del problema. Los estudiantes desarrollan habilidades en el uso de éstas al preguntarse cada vez que las usan por su utilidad, su fin y las relaciones entre el problema original y el transformado desde el punto de vista del proceso de resolución. Algunas herramientas heurísticas son: considerar un caso, analogía aclarada, división del problema en 
La idea de heurística en estudiantes para profesor de matemáticas Alvaro Arturo Sanjuan | Edna Paola Fresneda | Aseneth Gutiérrez | Oscar Leonardo Pantano

partes, el paso al contrarrecíproco, la introducción de una figura auxiliar, entre otras.

Por su parte las destrezas heurísticas no son meras formas adecuadas de trabajo, sino que sirven para descubrir. No tienen el carácter de transformación de las herramientas. Éstas son examinadas desde su uso para representar al resolver un problema. Su potencial heurístico está dado por la acción de descubrir ya sean relaciones, diferencias, semejanzas entre otras y por permitir el cambio de registro para comprender mejor el contenido del problema. A su vez, pueden ser enseñadas a través de un contenido matemático corriente. Algunos ejemplos de éstas son: hacer una tabla, un gráfico, utilizar una notación adecuada, entre otras.

Finalmente, las sugerencias heurísticas señalan una dirección de trabajo que permite avanzar en el proceso, sin hacer referencia a un procedimiento concreto para ello. Una de sus funciones se basa en desencadenar el uso de herramientas heurísticas y búsqueda de conocimientos previos teniendo en cuenta rasgos del problema. Entre ellas tenemos: buscar un problema relacionado y las preguntas planteadas por Polya (1945) en su estudio, relativas al objeto matemático.

Estrategias metacognitivas. Están relacionadas con el monitoreo del proceso llevado a cabo para resolver un problema. Mediante este proceso el resolutor se hace consiente de las limitaciones y capacidades propias. Teniendo en cuenta esto (Schoenfeld, 1987) identifica tres categorías donde se presenta la metacognición.

Primero, el conocimiento y descripción acerca de nuestro propio proceso de pensar. Segundo, el control y la autorregulación, hace referencia a qué tan bien es capaz uno de seguir lo que se hace cuando se resuelve algún problema y qué tan bien se ajusta uno al proceso. Allí se incluyen las decisiones relativas a la ejecución de un plan, al uso de la información que se tiene para resolver un problema. Tercero, las creencias e intuiciones desde las ideas acerca de las matemáticas implícitas en el trabajo matemático y la forma en que éstas se relacionan o se identifican con la manera de resolver problemas.

\section{Metodología}

La metodología consiste en una investigación en diseño o experimento de enseñanza de seis meses. Para ello, se realiza el diseño de un ambiente de aprendizaje que contempla los elementos teóricos de la investigación. Luego del diseño se pone en marcha el experimento y se rediseña hacia un mejorameinto progresivo de un diseño propio del proceso de instrucción. De esta forma, es constantemente revisado y modificado (Collins, et. al. 2004).

\section{Fase de diseño}

El diseño se realizó teniendo en cuenta elementos de la teoría cultural de la objetivación. Los elementos críticos del diseño fueron los siguientes. 1) los estudiantes discutían en pequeños grupos de trabajo o comunidades de aprendizaje su producción; 2) los estudiantes llevaban un cuaderno del resolutor donde consignaban su actividad matemática; 3) los libros de texto eran introducidos por el profesor para buscar un contenido específico en el momento requerrido; 4) el profesor discutía con los estudiantes los significados presentados por la comunidad de aprendices, teniendo en cuenta los significados atribuidos por la comunidad de matemáticos.

EI problema. El problema es parte fundamental del diseño, pues se trata de la empresa en torno a la cual trabaja la 
comunidad de aprendices. El enunciado del problema consistió en una afirmación matemática y los estudiantes debían discutir alrededor de ella para producir preguntas, conjeturas y justificaciones a las conjeturas. El enunciado del problema es el siguiente: "Sea $A$ el conjunto de los números en el intervalo $[0,1]$ cuya expansión decimal es de sólo 0, 5 y 9».

\section{Fase de implementación}

El experimento de enseñanza tuvo una duración de seis meses en el curso Problemas del Continuo que tiene como propósito tematizar la demostración en un contexto de continuidad de reales. Este curso a su vez hace parte del Proyecto Curricular de Licenciatura en Educación Básica con Énfasis en Matemáticas de la Universidad Distrital Francisco José de Caldas de la ciudad de Bogotá.

Los datos recogidos provienen de las videograbaciones de clase, las intervenciones en el sistema de aulas virtuales Moodle y los cuadernos de los estudiantes. Se escogió de manera aleatoria un grupo de cinco estudianes: Loly, Ángela, Katherine, Cindy y Jonath. Se llevó un registro de todas las clases y los datos se toman a partir de las transcripciones de las videograbaciones tomadas de la actividad matemática de los estudiantes.

\section{Fase de análisis}

El análisis de los datos se llevó a cabo teniendo en cuenta los planteamientos de Gavilán, García y Llinares (2007), apoyado en la elaboración de viñetas que son informes que vinculan información de diferentes fuentes. En éstas se integran inferencias e interpretaciones del investigador que se asocian a la evidencia de la práctica. Allí, se incluye el lugar cronológico en el que se desarrolla la actividad del estudiante, las tareas desarrolladas por el mismo, así como la organización del contenido matemático y las inferencias del investigador. Los resultados se presentan a continuación en tres viñetas.

\section{Resultados}

\section{La heurística como organizadora del trabajo (herramienta de negociación de la empresa)}

Los datos de esta viñeta provienen de la segunda y tercera sesión de clase, el grupo de estudiantes intenta comprender el conjunto con el propósito de plantear un problema a partir de la situación proporcionada por el profesor. Dicho problema debía apuntar a alguno de los siguientes conceptos: densidad, cardinalidad, convergencia u orden.

En la segunda sesión que tuvo lugar el 11 de Agosto, los estudiantes a partir de la lectura de la situación, buscan establecer cómo se comportan los números que pertenecen al conjunto. De esta forma, ejemplifican algunos números que pertenecen al conjunto, tratando de encontrar reglas o patrones generales.

\{1\} Katherine: Números cuya expansión es de sólo 0, 5 o 9 [Leyendo la situación].

\{2\} Ángela: O sea, es eso lo que uno dice, que tiene cero, cinco, o nueve [...] ¿Pero luego no decía, sólo ceros, sólo cincos, o sólo nueves?

\{3\} Katherine: Pero tú misma dijiste que pueden estar los tres. [...]

\{4\} Ángela: No, sería 0,05 o 0,059, ¿pero seguidos? ¿Podría ser? [...] 
La idea de heurística en estudiantes para profesor de matemáticas Alvaro Arturo Sanjuan | Edna Paola Fresneda | Aseneth Gutiérrez | Oscar Leonardo Pantano

\{5\} Katherine: Toca es tener en cuenta la combinación de los tres números para la expansión decimal.

\{6\} Ángela: Es que eso depende de cómo uno los coloque, porque uno puede decir digamos 0,05090 no sé qué, y ahí vuelve y se repite.

\{7\} Katherine: Por eso estoy diciendo lo de la combinación. [...]

\{8\} Cindy: Pero ¿ahí está diciendo que tiene que ser un número periódico? [...] Qué tal que sea un 0,3 , un 0,5, un 0,9 . [...]

\{9\} Ángela: Es decir, el cuento sería si es periódico o no, que es lo que dice Cindy.

\{10\} Loly: Yo creo, que toca delimitar, porque van a salir millones de millones de números.

\{11\} Cindy: Si, porque la expansión decimal puede dar un número decimal después de infinitos.

\{12\} Loly: Que no sean repetidos [... Digamos ¿cuántos números distintos hay en ese conjunto? Pero que no se repitan, o sea que no haya un 55 sino un 59, ¿me hago entender? [Escribe en su cuaderno]

\{13\} Cindy: Pero eso ya sería más por permutaciones ¿no? (corrigiendo) por permutaciones no, por combinaciones [...] Claro, porque se combinarían los dígitos.

\{14\} Jonathan: Pero uno para combinar ahí ¿qué hace?, sabiendo que se puede tener 0,5555 ta ta ta.

\{15\} Cindy: Pero no, porque se te está volviendo de infinitos números, entonces sabemos que pueden haber, los máximos por cada decimal ¿ves? que pude ser cero, cinco o nueve, entonces va a ser una combinación entre esos tres, ¿no?

Transcripción 1. Exploración inicial.

En las primeras líneas de la trascripción 1 (líneas 1-4) es posible identificar que los estudiantes a partir de la lectura de la situación, buscan comprender la expansión decimal de los números pertenecientes al conjunto dado. Aquí, se evidencia confusión sobre la implicación de la palabra "solo» en la definición de los elementos que pertenecen al conjunto (líneas 1-2). Por ello, el grupo considera dos posibles interpretaciones (líneas 2 y 5). La primera, es que en la expansión decimal de un elemento del conjunto solamente aparezca uno de los tres números dados. La segunda es que la expansión decimal esté compuesta a la vez por 0,5 y 9 .

El grupo toma algunos ejemplos (línea 4), que lo lleva a considerar un procedimiento sistemático para entender cómo se comportan los números del conjunto, la combinatoria. En consecuencia, se cuestionan acerca de que la expansión decimal sea o no periódica. En caso de ser periódica, el grupo asume que el conjunto se vuelve infinito. De acuerdo con esta implicación, se plantea una condición que permita restringir la cantidad de elementos del conjunto, asociada a la acción de no repetir los números en la expansión decimal. Esto los lleva nuevamente a considerar la combinatoria como la estrategia óptima para establecerlos (línea 7).

El grupo de estudiantes se vale continuamente de casos específicos para aclarar y dar validez a los planteamientos relativos a la expansión decimal y a no repetir números en ésta (líneas 4 y 8). Así, el grupo empieza a configurar su empresa, la cual se enfoca hacía la cantidad de elementos del conjunto 
(línea 12), asumiendo el hecho de que los números que conforman la expansión decimal no pueden repetirse. En la misma sesión de clase, el profesor interviene al ser buscado por los estudiantes, que se encuentran confundidos respecto al tema al que debe apuntar la pregunta. El profesor les menciona los distintos temas que se pretende abordar en el curso, para que sea posible centrar el trabajo.

\{1\} Cindy: Mire profe, decíamos que cuántos posibles decimales habrían dentro de $[0,1]$ sin que se repitan dígitos en un decimal ¿me entiende?

\section{\{2\} Profesor: ¿Cuántos? [...]}

\{3\} Cindy: Digamos 0,59, pero nunca puede haber 0,55 , o sea que tengan que tener la combinación de estos.

$\{4\}$ Katherine: Que los tres tengan que estar presentes en la expansión decimal. [...]

\{5\} Profesor: Pues en principio es una pregunta que podría servirles, pero debe ir apuntada a cuestiones de orden.

\section{\{6\} Loly: ¿O sea a comparar?}

\{7\} Profesor: [...] por ejemplo, hacia cuestiones de densidad, ¿qué quiere decir densidad? Que entre dos números sin importar cómo sean, yo puedo encontrar otro, o cosas así. Convergencia, hacia donde se acerca, ¿cierto? Eh... cardinalidad, más o menos lo que se están preguntando es decir ¿cuántos hay?, lo que pasa es que no nos interesa tanto cuántos hay en un número finito sino cuántos hay de manera infinita, o algo así, ¿sí? [Se retira] [...]

\{8\} Ángela: Cuando uno habla de orden ¿de qué está hablando?
\{9\} Loly: Cuál es más grande que.

\{10\} Ángela: Bueno listo, ¿qué es convergencia? [...] Ni idea. Es decir, lo único que sabemos es ¿qué es orden? Y ¿qué es cardinalidad? [...] ¿Entonces vamos a comparar las cantidades, para trabajar algo de orden?

\{11\} Jonathan: Por eso, pero cuando habla de expansión decimal es infinito.

\{12\} Katherine: Pero no necesariamente vamos a decir cuánto.

\{13\} Loly: Eso, porque cuando vamos contando entonces estamos encontrando más chiquitos. [...]

\{14\} Katherine: Digamos 0,59 ¿el anterior a ese cual sería? No se podría poner 0,58.

\{15\} Cindy: 0,50.

\{16\} Loly: ¿0,50? Y por qué no 0,55 y después 0,50 [señalando]

Transcripción 2. Intervención del profesor.

Los estudiantes deciden preguntarle al profesor acerca de los temas sobre los que podría plantearse el problema, puesto que él no considera que la combinatoria de números finitos les permita rápidamente entender asuntos del continuo numérico (línea 7). De acuerdo con esto, el profesor plantea a los estudiantes el hecho de que buscar el número de elementos en un caso finito no contribuye fuertemente al objetivo del curso. Aunque se podría utilizar inicialmente con el fin de trabajar con cuestiones de orden (línea 5).

Además, sugiere otros conceptos claves en el desarrollo del curso, sobre los cuales podrían trabajar (línea 7): densidad, cardinalidad y 
La idea de heurística en estudiantes para profesor de matemáticas Alvaro Arturo Sanjuan | Edna Paola Fresneda | Aseneth Gutiérrez | Oscar Leonardo Pantano

orden. Siguiendo las sugerencias hechas por el profesor, los estudiantes empiezan a cuestionarse acerca de estos objetos matemáticos (líneas 8-10). Sin embargo, deciden apuntar su trabajo hacia el orden, dado que es una noción sobre la cual sienten que tienen más dominio (línea 10), asemejándolo a la comparación entre números.

No obstante, surge nuevamente el infinito representando una gran dificultad en el desarrollo de su proceso de resolución (líneas 11-13). Finalmente, el grupo hace uso de un caso particular para empezar a establecer dicha comparación. (línea 14). De esta forma, la densidad y la comparación se convierten en temas implícitos en su trabajo (líneas 14-16). Así, comparan los números 0,59 y 0,50 y encuentran un tercer número entre los dos 0,55.

En el siguiente fragmento de transcripción, se evidencia, cómo la comparación de los elementos del conjunto se convierte en una estrategia, con el fin de establecer una relación de orden entre ellos. De esta manera, el grupo de estudiantes valiéndose de ejemplos, plantea una conjetura sobre el máximo del conjunto. Para ello, una de las estudiantes acude a las elaboraciones realizadas por compañeros de semestres anteriores vinculados al mismo espacio de formación, las cuales son tomadas del aula virtual del curso en el sistema Moodle.

\{1\} Loly: Mira, en la página de continuo [refieriéndose al aula virtual de moodle] había un ejemplo que decía: ¿qué número es más grande 0,444444 ó 0,474747 si tienen los mismos dígitos? Entonces ahídecía que después de la coma el cuatro y el cuatro eran iguales, y ¿cuál es el más grande el 7 o el 4? [Señalando en el cuaderno] [...]

\section{\{2\} Ángela: El 7.}

\{3\} Loly: Entonces, éste era más grande. [Señalando el cuaderno] [...]

\{4\} Ángela: Entonces nosotros habíamos dicho 0 coma y aquí hay 44 y 47, entonces como 47 es más grande que 44, entonces éste es el más grande [...] ¿cuál es el número más grande que se podría formar en la expansión decimal?

\{5\} Jonathan: Si, ¿cuál es el número más grande entre 0,5 y 0,005? [...]

\{6\} Ángela: Mire si 0,5 es igual a 0,05 al dividir 0,5 entre 0,05 nos tiene que dar 1. Si no nos da 1, entonces no es igual. [Lo dice con euforia]

\{7\} Profesor: Bueno ¿qué criterio tienen para definir eso? [Refiriéndose a la relación de orden establecida]

\{8\} Loly: Entonces lo que yo decía que estaba en la página de continuo era: él [refiriéndose al compañero del curso anterior] miraba después del cero, si estos dos eran iguales, después de la coma éste es más grande que éste, ¿cierto?

\{9\} Profesor: Ajá ¿Cómo es el criterio? Si es el más grande se compara término a término. Si el primero del uno es más grande ¿qué pasa?

\{10\} Loly: Éste es mayor. [...]

\{11\} Profesor: Entonces, éste es igual a éste [refiriéndose a los primeros dígitos de la posición decimal de los números], entonces no tenemos problema, éste es igual a éste, todavía no, pero éste es mayor que éste [refiriéndose a los primeros dígitos de la expansión decimal que difieren]. Entonces, no me importa lo que hay de aquí para allá, porque éste es el más grande, 
otra manera es restando ¿cómo sería restando? Y ¿que debería dar?

\{12\} Loly: Positivo. [...]

\{13\} Profesor: Entonces hay varios criterios para ver eso. Entonces esas preguntas van en relación ¿a qué?

\section{\{14\} Loly: A ¿cuál era más grande?}

\{15\} Profesor: Cuando ustedes preguntan ¿cuál es el más grande que hay? entonces ahí hay algo relacionado con el orden. ¿Podría precisar la pregunta? [Pausa muy larga] [...]

\{16\} Profesor: $Y$ ahí están trabajando el orden de forma natural, entonces la pregunta puede ir encaminada a situaciones relativas al orden, ¿qué situaciones hay relativas al orden?, ¿cuál es el más pequeño? ¿Cuál es el más grande? ¿Cuál es el ínfimo? ¿Cuál es el supremo? [...]

\{17\} Loly: ¿Será que el 0,999999 es el último?

\{18\} Profesor: Por ejemplo, ¿cuál es el más grande del conjunto? ahí ya tienen la pregunta. [...]

\{19\} Cindy: Una pregunta podría ser demuestre que el número mayor de la expansión decimal es 0,99999. [Le dice a Ángela]

\{20\} Jonathan: Si el grande lo comenzamos de aquí para allá, entonces el pequeño será de allá para acá, tendría uno que devolverse. [...]

\{21\} Cindy: O sea la pregunta podría ser demuestre que 0,9 periódico es el número mayor de la expansión y encuentra el número menor de la misma expansión.

Transcripción 3. Planteamiento del problema.
Con el propósito de encontrar una estrategia que le permitiera a la comunidad comparar dos números decimales y establecer cúal es mayor, Loly se apoya en producciones de un estudiante de un curso anterior. A partir de esto, ella identifica una estrategia que consiste en comparar cada una de las cifras decimales de dos números (línea 1). Lo anterior, le permite establecer una relación de orden entre distintos elementos del conjunto.

Así, surge en el grupo la destreza heurística, comparación, que lleva a la consideración de casos específicos los cuales inicialmente toman números que no pertenecen al conjunto dado (líneas 1-4). Esto se debe al hecho de que se está intentando comprender el procedimiento y darle sentido dentro del proceso de resolución. A pesar de que no se trata del mismo conjunto, Jonathan logra establecer una posible pregunta a partir de la interacción dada en la comunidad (línea 5).

En este contexto, el grupo inicia una exploración sobre el conjunto tomando casos particulares (líneas 5-6), con el propósito de darle más solidez a la pregunta planteada. Es posible identificar que los estudiantes proponen una nueva estrategia, la división, que les permite establecer relaciones de orden entre elementos del conjunto (línea 6). Por otro lado, en la línea 11, el profesor les sugiere a los estudiantes otro criterio para el establecimiento de la relación de orden entre los elementos del conjunto $A$, de manera que noten que hay más de un criterio para comparar números. A partir de ello, él ayuda a los estudiantes a reconocer que el orden es la temática sobre la que giran las ideas y procedimientos desarrollados (líneas 13-16).

Por tal razón, los estudiantes interactúan construyendo de manera grupal la pregunta que guiará su proceso de resolución. Allí es posible evidenciar que gracias a los criterios 
La idea de heurística en estudiantes para profesor de matemáticas Alvaro Arturo Sanjuan | Edna Paola Fresneda | Aseneth Gutiérrez | Oscar Leonardo Pantano

puestos en juego Loly y Cindy logran reconocer que 0,9 periódico es el número mayor de dicho conjunto (líneas 17-19). Lo que permitiría afirmar que el grupo ha ganado comprensión sobre el conjunto dado. A su vez, en la línea 20 , se hace patente que Jonathan logra establecer una analogía, es decir, un procedimiento aplicable al problema de encontrar el elemento más pequeño del conjunto. A partir de esto Cindy plantea el problema recogiendo las ideas planteadas en la interacción que se llevó a cabo en la comunidad de aprendizaje (línea 21).

En resumen, es posible afirmar que existen tres aspectos importantes a la hora de identificar cómo las heurísticas ayudan en la construcción de una ruta en el abordaje y en el ataque de la situación en el proceso de resolución. En primer lugar, las heurísticas contribuyen a la comunidad de aprendizaje a familiarizarse con la situación puesto que es un elemento que permite dotar de sentido la empresa. Lo anterior hace referencia al hecho de que la situación propuesta por el profesor, al vincular aspectos tan diferentes (convergencia, densidad, cardinalidad, orden, entre otros), genera distintas posibilidades de abordaje.

De esta forma, la comunidad debe negociar no sólo conceptos, sino además procedimientos que la llevan a una interpretación compartida de la situación. Mediante lo anterior, se empieza a consolidar un quehacer común que permite organizar el trabajo de la comunidad.

En segundo lugar, el descubrimiento de nuevos conceptos, procedimientos y características de la situación. Este descubrimiento se hace posible a través de la consideración de casos, que a su vez permite encontrar patrones y regularidades que lleven a una generalización. Adicionalmente, el uso de ejemplos de manera sistemática es un escenario para el surgimiento de nuevos conceptos que pueden o no ser explícitos para la comunidad.

Así, la herramienta heurística particularización ayuda a que el grupo se apropie de la situación a partir del reconocimiento de características de ésta que no son explícitas pero que son importantes en el proceso de resolución.

La comunidad a través de la interacción social logra vincular diferentes formas de proceder que le permiten ganar más solidez sobre los argumentos puestos en juego. De manera análoga, la vinculación de los procedimientos implica la complejización de los objetos, pues permite a la comunidad de aprendizaje percibir aquellos rasgos esenciales que los componen. Así, los estudiantes están en lugar de reconocer que los objetos matemáticos poseen diversas maneras de ser expresados de acuerdo con su contenido conceptual.

Finalmente, las heurísticas permiten no sólo la consolidación de la empresa sino además el surgimiento de nuevos conceptos que influyen en el establecimiento de una ruta. Estos conceptos son el resultado de un proceso de negociación que tiene lugar dentro de la comunidad de aprendizaje, de tal forma que la empresa conjunta no es impuesta. Es posible afirmar que las heurísticas desempeñan un papel fundamental en la delimitación grupal de la empresa (o problema) y por ende en la organización del trabajo en el proceso de resolución.

\section{La heuristica como reorganizadora del trabajo}

De la tercera a la quinta sesión de clase los estudiantes buscan delimitar el problema tratando de encontrar el número más pequeño del conjunto. De esta forma, 
intentan convertir el problema de encontrar (encontrar el máximo) en uno por demostrar (demostrar que 0,9 es el máximo del conjunto). En este contexto, se enfrentan con un hecho que les cambia la conjetura inicial sobre el máximo del conjunto. En la sesión del 13 de agosto el grupo trata de plantear una conjetura sobre el elemento más pequeño del conjunto. En este momento los estudiantes se encuentran con un hecho de gran importancia, que hace referencia a que todo número natural tiene expansión decimal infinita de ceros. Sin embargo, no son conscientes de cómo este hecho afectará su trabajo.

\{1\} Loly: Bueno, cuál es el más pequeño del conjunto $0,0 \circ 0,5$. [...]

\{2\} Cindy: Pues cero, en eso quedamos.

\{3\} Katherine: Es que no sabemos si es $0,0 \circ 0,5$.

\{4\} Loly: ¿Mejor dicho 0,0 es un número? o ¿es igual a cero? o sea que no tiene expansión decimal, ¿si tiene?

\{5\} Katherine: Pero es cero.

\{6\} Loly: No, por eso te digo que todos los números naturales tienen expansión decimal infinita de ceros, o sea dos es igual a 2,0 periódico.

\{7\} Katherine: O sea, estás tomando estos como iguales y que estos números si existen [señalando]

$\{8\}$ Loly: 0,0 es igual a 0 .

\{9\} Jonathan: Tú no colocas cero.

\{10\} Ángela: [...] Dijimos que todos los números enteros después de la coma tienen ceros infinitos, entonces digamos 1,0 infinito es igual a 1 y así entonces 0,0 infinito, bueno periódico sería cero [escribiendo]

\section{\{11\} Jonathan: Cero.}

Transcripción 4. Delimitación de la situación problema.

El grupo de estudiantes tratando de convertir su pregunta inicial sobre cuál es el mínimo del conjunto, en una conjetura considera dos casos posibles para éste. Así, en las tres primeras líneas de la transcripción 4 se muestran los casos específicos tomados por el grupo, 0,0 y 0,5. A partir de ello entran en discusión acerca de si 0,0 es un número o no (líneas 4, 5, 8). El grupo establece una negociación que les permite notar que todo número natural tiene expansión infinita de 0 , mostrando un ejemplo específico de este hecho (línea 6).

Sin embargo, la discusión se retoma dado que no todos los integrantes del grupo están de acuerdo con dicha afirmación (líneas 4 y 7). Lo anterior, cuestionándose siempre sobre los mismos aspectos: la existencia de 0,0 , si éste es un número, si es igual que 0 , entre otros. Todo ello mediado por la consideración de dos casos específicos: 1,0 y 2,0 (líneas 6 y 10). Aunque Ángela plantea el caso de que 1 tiene expansión infinita de ceros, el grupo no es consciente de que éste hecho puede alterar la conjetura inicialmente planteada con respecto al número mayor del conjunto.

Posteriormente, Ángela evidencia el hecho de que si 1 tiene expansión decimal infinita de ceros la conjetura planteada inicialmente puede verse afectada, tomando consciencia de las implicaciones que esto tiene sobre el trabajo a desarrollar. Así, el grupo con ayuda del profesor se cuestiona sobre la relación entre 0,9 periódico y 1 , considerando la existencia de diversas formas de argumentar matemáticamente. 
La idea de heurística en estudiantes para profesor de matemáticas Alvaro Arturo Sanjuan | Edna Paola Fresneda | Aseneth Gutiérrez | Oscar Leonardo Pantano

\{1\} Ángela: Es que nosotros habíamos partido del hecho de que 0,0 periódico era el menor, porque todos los números enteros tienen una expansión decimal de cero, pero entonces si nosotras decimos eso con el cero, entonces también lo podríamos decir con él uno, porque el uno también podría tener expansión decimal de cero sino que no la utilizamos, entonces el mayor número no sería 0,9 periódico sino que sería 1, [...] 1,000

\{2\} Profesor: Ok, ¿cuál es la relación entre 0,9 periódico y 1 ?

\section{\{3\} Loly: Que crece pero nunca va a ser 1}

\section{\{4\} Ángela: 0,9 periódico es menor que 1}

\{5\} Profesor: ¿0,9 periódico es menor que uno?, Ok, [...] lo primero que van a hacer es mirar el número en fraccionario de 0,9 periódico, en relación con el 1, y si el uno pertenece al conjunto y llegara a ser más grande que 0,9 periódico que habría pasado con la conjetura de ustedes

\{6\} Katherine: Que era falsa

\{7\} Profesor: Si llegara a pasar otra cosa, entonces...

\{8\} Ángela: ...entonces podríamos seguir con la sucesión [...]

\{9\} Ángela: 0,9 periódico es igual a 1, según la conversión que hicimos [...] pues si 0,9 periódico es igual a 1 entonces nuestra conjetura seguiría bien

\{10\} Loly: no porque el mayor número sería 1

\{11\} Ángela: pero si 0,9 periódico es igual a 1 en fracción, entonces 0,9 periódico es igual a 1 ¿no? [...]
\{12\} Profesor: Mire que hay una cosa en la que estamos metidos, que 0,9 periódico es igual a 1, o que 0,9 periódico es menor que 1, [pausa larga] ¿sí? si es posible que 1 sea 0,9 periódico, tendrían que entrar a explicar por qué, porque eso se está volviendo crucial para la conjetura de ustedes, y isi esto es menor estrictamente que 1 pues al igual se les volvería falsa?, cierto, y tumbaría la conjetura, entonces tendrían que mirar, [pausa] si ustedes se van por acá [señala cero, nueve periódico] y resulta que esto es falso pierden todo el trabajo de aquí para adelante. Si me hago entender [...] pero si ustedes asumen esto [0,9 periódico el mayor que 1] y eso es falso, entonces perderían el trabajo de aquí en adelante, pues puede ser esto o esto pero no pueden ser las dos cosas, por lo que decíamos de la ley de tricotomía hace rato, o son iguales o el uno es menor que el otro pero no puede pasar que tantas cosas se estén cumpliendo al tiempo, ¿entonces cual es? Es esto o es esto. [...]

\{13\} Ángela: Entonces nos toca explicar eso. Porque uno no puede pasar por eso sin saber por qué.

\{14\} Profesor: [...] o ustedes mismos, dar razones de por qué... ¿cómo pueden dar razones? Ustedes pueden hacer sus propios patrones, sus propios registros, sus propios ejemplos, intentando justificar hacia un lado o hacia el otro.

\{15\} Ángela: 0,9 periódico sería el máximo del conjunto A ¿cierto? [Ángela escribe en el cuaderno mientras dice en voz alta lo que escribe][...] surgió una pregunta, 0,9 periódico va a ser igual a 1 ? 0 ¿ 0,9 periódico es menor de 1?

Transcripción 5. Inestabilidad de la conjetura inicial. 
La comunidad de aprendizaje reconoce la herramienta heurística analogía evidenciando que las nociones y procedimientos utilizados para encontrar el máximo del conjunto pueden ser utilizados de manera similar para encontrar el mínimo (línea 1). Los estudiantes se hacen consientes de cómo el aspecto identificado se vuelve relevante para la conjetura planteada. Dicho aspecto está relacionado con que todo número entero tiene expansión infinita de ceros, hecho que antes no había sido considerado con todas sus implicaciones.

De acuerdo con lo anterior, Ángela logra considerar una nueva conjetura la cual hace referencia a que 0,9 periódico no es el máximo, sino que es 1,0 periódico. Dado que este hecho se vuelve crucial en el proceso de resolución, el grupo decide acudir al profesor para que él les ayude a orientar su trabajo. El profesor busca que inicialmente la comunidad establezca la relación existente entre estos números (líneas 2-5). La idea de dicha relación por parte de los estudiantes apunta a que 0,9 siempre va a ser menor que 1, aunque se acerque infinitamente. Sin embargo, el profesor les sugiere un procedimiento que les permita establecer la relación ya mencionada.

Es posible inferir que el profesor busca que sean los estudiantes, mediante su propia acción quienes noten la diferencia o igualdad entre 0,9 periódico y 1 . (línea 5). A su vez cuestiona a los estudiantes acerca de si 1 pertenece al conjunto dado, puesto que ellos no tienen seguridad de la pertenencia de este elemento y les hace considerar la relevancia de establecer una conjetura sólida con respecto al hecho encontrado. Es decir, los estudiantes deben reconocer que sus acciones afectaran la conjetura inicialmente planteada (líneas 5-12).

A partir de la interacción dada en el grupo y la conversión de 0,9 periódico a su corres- pondiente número fraccionario Ángela logra establecer que la relación existente entre 0,9 periódico y 1 es de igualdad (línea 9). Ella afirma que la conjetura inicial no se vería afectada, frente a lo que se genera una discusión pues Loly plantea que a partir de la igualdad lo correcto sería conjeturar que 1 es el máximo del conjunto (línea 10). De este modo, la comunidad de aprendizaje continúa con la discusión por lo cual deciden acudir nuevamente al profesor.

Posteriormente, el profesor en su intervención hace énfasis en los dos caminos que han surgido en el proceso de resolución y en la importancia de escoger adecuadamente uno de estos (línea 12). Teniendo en cuenta que el proceso se vería afectado a partir de la toma de decisiones apresuradas. Además, se hace un cuestionamiento acerca de la pertinencia del procedimiento utilizado para establecer la relación de orden entre 0,9 periódico y 1 (línea 14), dado que este procedimiento finalmente sólo es un algoritmo.

En este momento, se hace evidente que el grupo deja de lado momentáneamente su conjetura inicial, para dar paso a un problema previo que les permita identificar si han falseado o no su conjetura. Así, el grupo reconoce que su proceso de resolución ha tomado un nuevo rumbo, a partir de la consideración de dos posibles caminos de acuerdo con lo mencionado por Ángela (línea 15).

En conclusión se puede observar que las herramientas heurísticas consideración de casos y analogía posibilitan que se explote el potencial heurístico, es decir, que se descubran nuevos conceptos y procedimientos que permiten visualizar rutas de resolución nuevas. Entre dichos conceptos destacamos principalmente el hecho de que todos los números enteros tienen expansión decimal infinita de ceros. 
Este descubrimiento genera inestabilidad en las ideas y acciones del grupo, dado que se propicia el cuestionamiento de la conjetura planteada inicialmente por la comunidad de aprendizaje. Cuando la comunidad de aprendices se desestabiliza, siente la necesidad de adaptarse a las nuevas condiciones que exige el aprendizaje. Resultado de éste, se produce el significado, fruto de las interacciones con los elementos inmersos en el mundo (Wegner, 2001).

Teniendo en cuenta lo anterior, el reconocimiento de nuevos elementos implícitos en la situación propicia la transformación del problema inicial en otro. Así, la transformación del problema obliga a la comunidad a desarrollar nuevamente un proceso de participación y negociación, en el que se establecen nuevas rutas de abordaje que se convierten en un repertorio compartido, dándole sostenibilidad a la empresa común.

Finalmente, es posible afirmar que al ser el aprendizaje una estructura social emergente y perturbable debe tener una capacidad de adaptación. Esta susceptibilidad al cambio hace que el uso de las heurísticas en el proceso de resolución posibilite descubrimientos, que implican la constitución de un nuevo quehacer que es común y que desencadena caminos de actuación frente al problema que son diferentes y novedosos.

\section{La heuristica asociada a la elaboración de significado}

El grupo de estudiantes en el intento de demostrar que 0,9 periódico es el máximo del conjunto, inicialmente construye una demostración formal basándose solamente en elementos teóricos. Acuden así al profesor con el fin de que él la valide, siendo éste quien les ayuda a reconocer la falta de comprensión y dominio de diversos conceptos y procedimientos involucrados, además de la relación de éstos con la situación. En otro momento, tratando de demostrar esta misma conjetura los estudiantes recurren a tres procedimientos propios con el fin de establecer criterios que posteriormente se convertirán en argumentos para la demostración.

En las sesiones de clase del 13 y 18 de agosto, el grupo está intentando demostrar formalmente la primera conjetura propuesta. Es decir, que 0,9 periódico es el máximo del conjunto, haciendo uso de referentes teóricos sobre relaciones de orden, a partir de sus propiedades (reflexiva, antisimétrica y transitiva). Lo anterior lleva al grupo a cuestionarse con ayuda del profesor, sobre diversas nociones y conceptos, tales como: qué tipo de relación de orden se establece entre los elementos del conjunto, qué es una relación de orden parcial, entre otras. Así, se hace evidente que los estudiantes no tienen claridad sobre estos aspectos, por lo tanto se dificulta vincularlos a la situación.

\{1\} Ángela: Entonces buscamos un referente teórico para demostrar en general que 0,9 es el mayor del conjunto. [...] Eso es de cardinalidad y de orden, pero es que estamos buscando todo eso de orden, porque el profesor dijo que era de orden.

\{4\} Jonathan: Es que aquípodemos ver que es una relación de orden, pero partiendo de una relación binaria.

\{3\} Ángela: Entonces deberíamos buscar eso, y buscar lo que dice el libro respecto a una relación, listo [...] 0,9 es menor que el 0,9 periódico luego 0,9 no va a ser mayor que el otro, porque si decía si Bernardo está a la izquierda de Andrés, pues Andrés no podría estar a la izquierda de Bernardo bueno pues algo así. Bueno si Catherine es mayor que yo, entonces, yo no puedo ser 
mayor que Catherine [...] bueno pues esa la idea,.. Bueno pues demostrar eso.

\{4\} Loly: Bueno pues digamos que a y $b$ son números del intervalo. [...]

\{5\} Cindy: pero qué es lo que vamos a mirar, ¿qué b es mayor que $c$ ? o que se relaciona

\{6\} Katherine: pues yo estaba haciendo si a es menor que $b, y b$ es menor que $c$, pues $a$ es menor quec,

\{7\} Cindy: Pero a no es menor que $c$ ¿o sí? [...]

\{8\} Profesor: Bueno, entonces vamos por partes, lo primero cuál era la conjetura.

\{9\} Katherine: Eh, pues eran dos conjeturas, una es demostrar que 0,99 periódico es el más grande del conjunto y la otra que 0,0 periódico es el menor del conjunto, pero aquí estamos haciendo primero la del 0,99 periódico.

\{10\} Profesor: O sea que 0,9 periódico es el más grande del conjunto y esa es la conjetura de nosotros, entonces probamos reflexividad, antisimetría y transitividad [...] hay una relación de orden, bueno cuando uno prueba reflexividad, antisimetría, y transitividad está probando efectivamente que hay una relación de orden o un orden parcial, se llama así, listo. Ahora ¿no hay un orden en esta conjetura que ustedes tienen?

\{11\} Loly: Primero va el cero, luego el cinco y luego el nueve. [...]

\{12\} Profesor: Ok entonces aquí está probando que 0,9 periódico es más grande que los de expansión finita 0,9, pero yo pensé que ustedes estaban trabajando en que 0,9 es el más grande del conjunto.
\{13\} Katherine: Si.

\{14\} Profesor: Entonces este orden de acá es reflexivo, antisimétrico, y transitivo. Pero qué estamos probando.

\{15\} Loly: Que hay un orden.

$\{16\}$ Profesor: Hay varias clases de orden, una se llama orden parcial y otra se llama orden total [...] aquí hay un ejemplo que es parcialmente ordenado, que no va en filita y no tiene último, entonces qué es lo que está pasando, que sea orden parcial no garantiza la existencia del máximo, ustedes ya tienen orden, que es el orden ¿de quién? ¿Cuál es la relación de orden hay?

\{17\} Loly: Que hay un cero, luego hay un cinco y luego hay [...]

\{18\} Profesor: Pero eso sería de los decimales que podemos usar, pero por ejemplo, si yo quiero comparar 0,50 con 0,59 ¿ahí no hay un orden?

Transcripción 6. La inestabilidad de una primera demostración.

El grupo de estudiantes tratando de demostrar que 0,9 periódico es el máximo del conjunto, hace uso de referentes teóricos evidenciando que la teoría sobre relaciones de orden es un posible camino de ataque de la situación problema. De esta forma, los estudiantes intentan establecer una conexión entre la conjetura y las relaciones de orden, tomando un ejemplo de éstas (líneas 1-3). Sin embargo, es posible identificar que los estudiantes no logran establecer esa conexión.

Debido a ello, dejan a un lado dicha acción para centrar su trabajo en intentar establecer una demostración formal, para lo que se definen ciertos elementos $a, b$ y $c$ 
La idea de heurística en estudiantes para profesor de matemáticas Alvaro Arturo Sanjuan | Edna Paola Fresneda | Aseneth Gutiérrez | Oscar Leonardo Pantano

y algunas relaciones entre éstos, por ejemplo, que $a$ es mayor que $c$ (líneas 4-7). Allí se hace evidente que los estudiantes no vinculan la demostración que pretenden construir con los elementos propios de su situación. En consecuencia, se da una discusión en la cual es posible observar que no hay suficiente claridad a cerca de lo que se pretende demostrar, es decir sobre la tesis de su demostración.

No obstante, Loly muestra su intento de demostración a la comunidad de aprendizaje, quienes deciden acudir al profesor para que valide la demostración realizada. De este modo, en la interacción que tienen lugar entre el grupo de estudiantes y el profesor, éste último intenta retomar la conjetura, siendo ésta el elemento a partir de la cual se desarrolla la demostración, además de las acciones realizadas por los estudiantes para construir esta última (líneas 8-10).

A partir de ello, surgen unos cuestionamientos del profesor hacia los estudiantes sobre la relación de orden que se puede evidenciar en el conjunto; frente a esto los argumentos dados por los estudiantes son muy débiles dado que no hay una amplia comprensión sobre los conceptos inmersos tanto en el conjunto como en las relaciones de orden. Así, el profesor ayuda a los estudiantes a entender el orden del conjunto por medio de la definición del concepto de orden parcial. De esta forma, él busca centrar el trabajo con respecto a la conjetura inicial, dado que en este momento (al inicio del curso) los estudiantes se encuentran alejados en la consecución de su objetivo (líneas 10-18).

Además se puede observar en las líneas restantes de la transcripción que el profesor se esfuerza por que el grupo de estudiantes note que en el proceso que están llevando se está dejando de lado aquello que se plantearon como problema inicialmente. Esto se hace evidente cuando el profesor les expresa que el hecho de que haya un orden parcial en el conjunto, no garantiza la existencia del máximo. Así, él espera que los estudiantes reconozcan que están siguiendo un camino equivoco, puesto que es diferente al que se trazaron inicialmente.

Es posible inferir que la desvinculación de los conceptos y procesos utilizados se debe al nulo uso de ejemplos, pues como se vio anteriormente éstos permiten no sólo la aparición de nuevos conceptos, sino además su comprensión mediante la consideración y aplicabilidad en el proceso de resolución. Por esta razón, los estudiantes pierden control del proceso al intentar vincular, de manera forzada, demostraciones formales. No obstante, el control del proceso ocurre cuando emplean heurísticas propias.

En la sesión del 25 de agosto, el grupo con ayuda del profesor está tratando de establecer como argumentos los procedimientos matemáticos utilizados para encontrar la relación existente entre 0,9 periódico y 1 . Así, encuentran tres criterios que les permiten afirmar con certeza que 0,9 periódico es igual a 1 .

\{1\} Profesor: Entonces se trata de intentar mostrarlo con argumentos matemáticos, como por ejemplo, criterios de diferencia ¿Qué otro criterio de que uno sea más grande que el otro puede haber? [...]

\{2\} Ángela: ¿Dividiéndolo no?, yo por acá alguna vez había hecho lo de la división. Es que miré si uno lo divide, y le da 1 es porque son iguales.

\{3\} Profesor: Pues entonces miren, una cosa que hicieron fue la división. 
\{4\} Cindy: La diferencia.

\{5\} Profesor: La diferencia, y hay otra cosa que hicieron ¿qué fue?

\{6\} Cindy: Pasar un número decimal a fraccionario [señala su cuaderno]

\{7\} Profesor: En los tres casos qué está pasando.

\{8\} Ángela: Pues que en éste nos da 1 [señala su cuaderno] En éste también nos da uno.

\section{\{9\} Cindy: ¿y en el otro?}

\{10\} Loly: Daba 0,0 infinito 1, en el de la resta.

\{11\} Profesor: Bueno pero es que esta resta es de un híbrido, pero como el híbrido no es posible entonces está restando también, si. Entonces la resta, ¿cómo es que da?

\{12\} Loly: Da cero.

\{13\} Ángela: Entonces si es 1.

\{14\} Profesor: Y el otro argumento que tenían era uno de conversión de racionales. ¿Hay otro argumento matemático que tengan por ahí para comparar los números?

\{15\} Ángela: Es que una vez dividimos el uno entre el otro, yo me acuerdo. Mírenla acá, bueno si tenemos que 0,5 y 0,05 son iguales podemos dividir 0,5 y 0,05 y nos tendrá que dar 1.

\{16\} Profesor: Si matemáticamente lo que les está dando, independientemente de la intuición, por distintos argumentos es que 0,9 periódico es 1, lo que quiero decirles es que, no entiendo por qué no entiendo, no me parece porque no me parece, o porque creo que no, no son argumentos matemáticos. [...]

\{17\} Ángela: Tenemos que llegar a un acuerdo entre todos.

\{18\} Loly: Ya hay un ajá, porque mire primero lo hicimos por diferencia pero no hay un híbrido, no existe en el mundo de las matemáticas. El otro es [...]

\{19\} Ángela: Conversión de decimal a fraccionario.

\{20\} Cindy: Nos da 1. La conversión nos apunta a que es 1 .

$\{21\}$ Loly: Si o sea a que son iguales.

\{22\} Katherine: Y lo de la división.

\{23\} Loly: Dividimos 0,9 periódico entre 1 y nos dio igual a 1. Entonces tenemos tres cosas, que nos dicen que es igual, ¿ya se convencieron?

\{24\} Ángela: Si, si, si.

(Ver esquema 1).

Transcripción 7. Construyendo argumento

Como se ve en la transcripción, a partir de la interacción dada entre el profesor y el grupo de estudiantes, estos últimos durante el proceso han desarrollado unos procedimientos propios que le han permitido enfrentarse a la situación. En este sentido, la intervención del profesor está encaminada a ellos noten este aspecto relevante en el proceso, con el fin de que se conviertan en criterios que permitan dar mayor solidez a los argumentos puestos en juego, para establecer la relación existente entre 0,9 periódico y 1 . A partir de esto, se pueden identificar tres procedimientos: la división, la resta y la conversión de un 
La idea de heurística en estudiantes para profesor de matemáticas Alvaro Arturo Sanjuan | Edna Paola Fresneda | Aseneth Gutiérrez | Oscar Leonardo Pantano

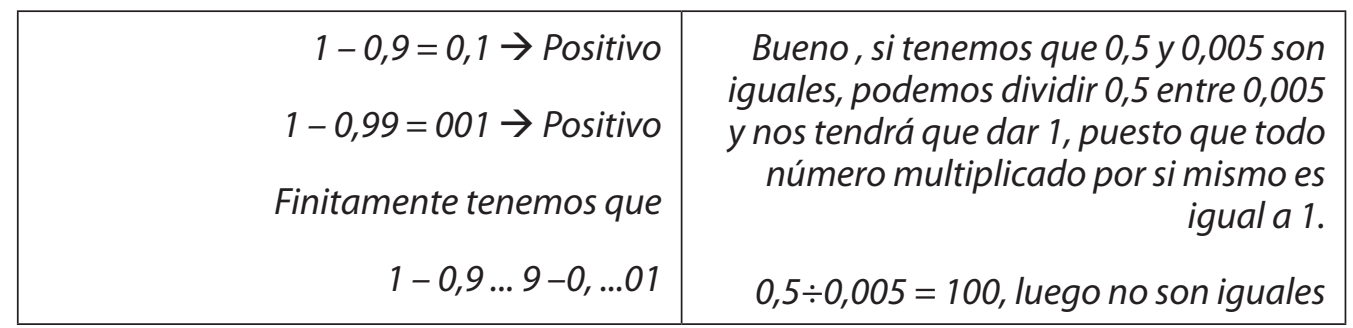

Texto 1 tomado del Cuaderno de Ángela (agosto 20 de 2009, página 19).
Texto 2 tomado del Cuaderno de Ángela (agosto 11 de 2009, página 6).

Esquema 1. Textos tomados del cuaderno de Ángela.

número decimal a un fraccionario, (líneas 2,4 y 14 respectivamente) y en los textos tomados del cuaderno de Ángela.

Es posible afirmar que este tipo de habilidades permitieron a los estudiantes no sólo desarrollar una ejemplificación sistemática, sino que a partir de ello lograron evidenciar claramente un hecho que inicialmente fue confuso y atascó su proceso de resolución. Éste hacía referencia a la relación de orden existente entre dos elementos del conjunto $(0,9$ periódico y 1$)$, pues este suceso en su momento generó dificultad en el quehacer del grupo. Así, mediante el descubrimiento y uso de estos procedimientos la comunidad logró establecer argumentos matemáticos que daba solidez a la conjetura. Además, se ve alcanzado uno de los propósitos en la intervención del profesor, quien buscaba que los estudiantes utilizaran argumentos matemáticos para validar sus acciones.

Buena parte de la atención y la reflexión se centra en los procesos de ejemplificación desarrollados por los estudiantes. El profesor solamente se encarga de establecer un puente entre los procesos desarrollados por el grupo, las matemáticas científicas y la situación particular sobre la que se está trabajando. Cabe mencionar que la interacción del grupo y el uso de ejemplos, le permite a la comunidad apropiarse de los conceptos y procesos puestos en juego en el ataque de la situación. A partir de ello, están en capacidad de dar cuenta tanto de la manera en que se está llevando el proceso, como de los elementos inmersos allí. Así, se posibilita que el grupo siga sin inconvenientes su ruta de resolución planteada y logre cumplir con el objetivo propuesto.

Por otro lado, es posible afirmar que el libro de texto es un artefacto que permite al resolutor el encuentro con la construcción histórica del objeto realizada por la comunidad científica. Además, éste moldea la actividad matemática del estudiante en tanto que plasma un contexto histórico-social determinado que le da forma y sentido. En consecuencia, el uso del libro de texto debe estar acompañado de una serie de acciones que le permitan al estudiante apropiarse de la actividad matemática, transformándola y aplicándola según sus necesidades.

Esas acciones cuando son desarrolladas en el proceso de resolución permiten identificar relaciones a partir de las cuales es posible consolidar argumentos. En este caso, éstas son procedimientos o heurísticas propias de los estudiantes tales como: división, 
resta y conversión de un número decimal a fraccionario, que se convirtieron en criterios que permitieron no perder la ruta que daba sentido a su empresa y el control heurístico del proceso.

La interacción social permite que las diferentes maneras de comprender el mundo se negocien y se complementen dando lugar a un repertorio compartido. Específicamente este repertorio se compone de la ejemplificación y de los tres procedimientos antes mencionados, los cuales se convirtieron en el elemento central del proceso de resolución. Éstos, dan lugar a un proceso de reflexión que permite a la comunidad de aprendizaje elaborar significados tanto de las acciones realizadas como de los objetos matemáticos puestos en juego.

Teniendo en cuenta los planteamientos de Puig (1996) tanto la ejemplificación desarrollada como las acciones utilizadas para la misma, se definen en términos de herramientas y destrezas heurísticas. Éstas, al acompañar el proceso de resolución permiten a la comunidad aprender haciendo, es decir, elaborar significados de conceptos y procedimientos a partir de su actividad propia. Ello se ve reflejado en el nivel de argumentación que se desarrolla para dar validez y rigor matemático al hecho de que 0,9 periódico es el máximo del conjunto $A$.

\section{Conclusiones}

Por medio de la interacción social la comunidad de aprendizaje logra relacionar modos de actuación diversos en el proceso de resolución, los cuales son denominados por Puig (1996) heurísticas. Éstas permiten la construcción de argumentos más sólidos y rigurosos, partiendo del hecho de que se trabaja con objetos matemáticos que exigen un cierto nivel de dificultad. De esta manera, la comunidad está en capacidad de reconocer las diversas características y usos de los objetos, a partir de la identificación de las distintas formas en que pueden ser expresados. Este reconocimiento se convierte así en un proceso de elaboración de significados.

En este sentido, las herramientas heurísticas pueden entenderse como un artefacto dado que fomentan el desarrollo de nuevas capacidades intelectuales. Esto, debido a que permiten percibir la inteligencia depositada en los objetos construidos social y culturalmente por generaciones pasadas. A lo largo de la historia, los objetos son marcados por la actividad de los individuos que los producen. Dicha actividad desencadena que esas formas de expresar el objeto se componga de elementos variados, relativos a la estética y la funcionalidad, entre otros, los cuales son propios de la cultura (Radford, 2006).

Reconociendo la consideración de casos como una herramienta heurística, ésta se convierte en un elemento constitutivo del proceso de resolución que posibilita que el estudiante reconozca las características y conceptos que son relevantes en éste. No obstante, aunque éstos no son explícitos en la situación promueven la elaboración de significados. A su vez, se debe tener en cuenta que estas acciones denominadas herramientas heurísticas dan lugar a la transformación y aplicación de la actividad matemática. Es decir, le permiten al estudiante apropiarse de dicha actividad matemática no sólo conceptual sino procedimentalmente, adaptándola a sus necesidades y dotando así de sentido todo lo que se hace. Además, las herramientas heurísticas se consolidan como un quehacer común a partir de la interacción social que tiene lugar en la comunidad de aprendizaje. 
La idea de heurística en estudiantes para profesor de matemáticas Alvaro Arturo Sanjuan | Edna Paola Fresneda | Aseneth Gutiérrez | Oscar Leonardo Pantano

El valor del uso de las herramientas heurísticas recae sobre el hecho del reconocimiento de su utilidad. Si a pesar de conocer dichas herramientas se desconoce cuál, cómo y cuándo emplearlas, el provecho de las mismas tampoco será reconocido. De acuerdo con esto, es preciso que la intervención del profesor, potencie en los resolutores el reconocimiento de la utilidad de dichas herramientas para que a su vez se ganen dominio frente a éstas.

Frente a ello, es posible afirmar que las heurísticas permiten la consolidación de la empresa y el surgimiento de nuevos conceptos, los cuales determinan una ruta de trabajo. Así, la empresa se convierte en el objetivo común del grupo de estudiantes. Tanto los conceptos, como la empresa, son fruto de un proceso de negociación que tiene lugar dentro de la comunidad de aprendizaje. Lo que sustenta la idea de que la empresa conjunta no es impuesta (Wenger, 2001). Es posible afirmar que las heurísticas desempeñan un papel fundamental en la delimitación grupal de la empresa y por ende en la organización del trabajo en el proceso de resolución.

Ahora bien, el aprendizaje es visto como una estructura social emergente y perturbable, la cual debe tener capacidad de adaptación. Es así, como al generarse desestabilidad la comunidad de aprendizaje busca ajustar su práctica a las nuevas condiciones que exige el aprendizaje. De este modo el encuentro entre los elementos del mundo y los individuos produce el significado (Wenger, 2001). Esta idea se refleja en la práctica cuando el uso de las heurísticas en el proceso de resolución posibilita descubrimientos que implican la constitución de un nuevo quehacer. Éste es común y permite desencadenar caminos de actuación frente al problema que son diferentes y novedosos.
En este punto, el grupo de estudiantes logra generar nuevas situaciones problema a partir de la situación propuesta por el profesor. A partir de lo anterior, es evidente como la herramienta heurística genera la definición y redefinición de la empresa, cuando es utilizada de manera adecuada.

A su vez, en el proceso de resolución el resolutor muchas veces se propone así mismo sugerencias heurísticas que le permiten avanzar. Sin embargo, dichas sugerencias pueden provenir en algunas ocasiones del profesor, lo cual permite que se fomente un ambiente de interacción. En éste se busca que los estudiantes construyan estrategias más sólidas que les permitan abordar y avanzar frente a la problemática propuesta (Puig, 1996).

Ahora bien, en el aula de clase vista como una comunidad de aprendizaje el profesor debe estar en lugar de reconocer y valorar la diversidad de los estudiantes, a través de las distintas formas de participación. Además, éste debe proponer tareas y problemas que permitan reconocer el saber como una construcción histórica y sociocultural no terminada. En este sentido, las situaciones problema deben ser lo suficientemente complejas para posibilitar la aparición de nuevas estrategias y nuevos aprendizajes. El profesor hace que se generen discusiones, al mismo tiempo que interviene en éstas solamente cuando es necesario (Radford, 2006).

A partir de esto, la intervención del profesor busca en diferentes momentos, promover en los estudiantes la habilidad de argumentar matemáticamente y de forma rigurosa tanto los procedimientos como los objetos usados durante el proceso de resolución, dejando de lado las intuiciones y posturas de autoridad. Por otro lado, el profesor interactúa con la comunidad de aprendizaje 
con el propósito de hacerle notar a los estudiantes que las acciones que se están realizando no corresponden al objetivo que se trazaron. Por lo que éste los cuestiona sobre la relevancia de sus actos y la manera misma de proceder frente a la situación.

Dado que el aprendizaje va más allá de la transmisión y repetición mecánica de conocimientos y procedimientos matemáticos, el objetivo de éste, es dotar de sentido los objetos, percibiéndolos por medio de la actividad propia del estudiante, la cual debe permitirle además, reconocer la expresividad del objeto de distintas formas conocidas por la humanidad. Es en este punto en donde se posibilita la formación de nuevas capacidades propias del individuo, las cuales están ligadas al desarrollo de ciertas acciones que permiten comprender el mundo (Radford, 2006).

Teniendo en cuenta la actividad real y las acciones desarrolladas por los estudiantes en términos de herramientas y destrezas heurísticas se posibilita la consecución de la empresa así como el dotar de sentido lo que se hace. Estas acciones al acompañar el proceso de resolución permiten a la comunidad de aprendizaje aprender haciendo, es decir, elaborar significados de conceptos y procedimientos a partir de su actividad propia. A su vez, mediante el uso de las heurísticas la comunidad se apropia de los conceptos y procedimientos vinculados en el proceso de resolución.

En los resultados se evidencia el uso de las heurísticas llevado a cabo en la práctica del grupo de estudiantes para profesor de matemáticas. Esto revela un conocer práctico. El reconocimiento explícito de las heurísticas se ve en la intervención de Ángela en el aula virtual Moodle el día 6 de septiembre cuando se le pregunta " $i Q u e ́$ sentido tienen las heurísticas en los problemas?»:
Yo creo que las heurísticas son importantes en el grupo porque principalmente uno siempre tiende a mirar lo que otros han hecho respecto a cierta investigación que tiene cierto parecido con los problemas matemáticos que planteamos, sin darnos cuenta de que nosotros podemos llegar a las respuestas partiendo de la realización de ejemplos que nos lleven a generalizar, encontrar patrones y regularidades cuando se miran las secuencias, utilizar diferentes representaciones y cambios de registro. Esto no quiere decir que uno deseche los avances que otros han tenido, pero si nos ayuda a no depender tanto de lo que otros han hecho sino que por medio de instrumentos propios se pueden llegar a conclusiones importantes.

Transcripción 8. Reconocimiento explícito de las heurística.

En esta intervención se ve, que según la teoría cultural de la objetivación, la toma de conciencia de la heurística va en el lugar de tener un dominio propio sobre los problemas. Asímismo, se ve vinculada principalmente a la idea de herramienta heurística, sobre la de destreza o sugerencia. La idea de heurística está asociada a la vivenciada en la práctica; pues como se evidencia en la transcripción, Ángela solo referencia las herramientas heurísticas vividas y deja de lado las no vividas como un problema simplificado o un problema general.

A partir de lo anterior, las heurísticas hacen referencia a las formas de hacer construidas y heredadas mediante el desarrollo históricocultural, es decir, pueden ser entendidas como objeto didáctico y no como objeto matemático, éstas moldean la actividad del individuo de unas maneras específicas y le posibilitan enfrentarse así a diferentes situaciones problema que requieren para su desarrollo un quehacer matemático, que pertenece a la vez al individuo y a la comunidad. 
De acuerdo con esto, las heurísticas también habitan en el mundo social, cultural e históricamente constituido (Radford, 2006). Es allí donde éstas y su utilidad se reconocen como un bien común, que surge de manera espontánea y se convierten en medios de la resolución de problemas y de la adquisición de conocimientos. Así, se hacen útiles en la práctica y se reconocen explícitamente como medios en el proceso de resolución, donde es posible hablar de cierto grado de reconocimiento, es decir, de un proceso social de toma de conciencia.

\section{Agradecimientos}

Nuestro especial agradecimiento al Centro de Investigaciones y Desarrollo Científico de la Universidad Distrital Francisco José de Caldas por la financiación y apoyo institucional brindado al este proyecto de investigación. Agradecemos también a Loly, Ángela, Katherine, Cindy y Jonathan por su colaboración y paciencia en el desarrollo de ésta investigación.

\section{Bibliografia}

Collins, A., Joseph, D. \& Bielaczyc, K. (2004). Design Research: Theoretical and methodological issues. The journal of the learning sciences. Número 13 (1), 15-42.

Gavilán, J., García, M. \& Llinares, S. (2009). Una perspectiva para el análisis de la práctica del profesor de matemáticas. Implicaciones metodológicas. Revista Enseñanza de las Ciencias, Número 25 (2), 157-170.
Duval, R. (1991). Structure du raisonnement déductif et apprentissage de la demonstration. Educational Studies in Mathematics 22 (3), 233-261.

Hemmi, K. (2008). Students' encounter with proof: the condition of transparency. ZDM Mathematics Education 40.413-426

Polya, G. (1965). Cómo plantear y resolver problemas. Editorial Trillas, México.

Puig, L. (1996). Elementos de Resolución de Problemas, Colección Mathema, Editorial Comares, Granada.

Radford, L. (2006). Elementos de Una Teoría Cultural de la Objetivación. Revista Latinoamericana de Matemáticas. (Número Especial) 103-129.

Sanjuán, A. \& Bonilla, M. (2008). Proyecto de Investigación: El Proceso de Demostración como instrumento de aprendizaje en la formación de profesores, Grupo Mescud, Colombia.

Santos, M. (2007). La resolución de Problemas matemáticos, Fundamentos Cognitivos, Editorial Trillas, México.

Simon, M. (1996). Justification in the mathematics classroom: A study of prospective elementary teachers. The Journal of Mathematical Behavior. 15 (1) 3-36

Wenger, E. (2001). Comunidades de aprendizaje: Aprendizaje, Significado e Identidad. Paidos. 